\title{
OPERATORS WITH POWERS ESSENTIALLY SIMILAR TO THOSE OF THEIR ADJOINTS
}

\author{
S. M. PATEL
}

\begin{abstract}
Let $T$ be an operator on a Hilbert space $H$. In the present note the following result is obtained:

If $T$ is an operator such that for some integers $p, q, S T^{*_{p}}=$ $T \cdot S+K$, where 0 is not in the essential numerical range of $S$, and $K$ is compact, then for any complex number $\lambda$ in the essential spectrum of $T, \lambda^{*}{ }^{*}=\lambda^{a}$.
\end{abstract}

Williams [10, Theorem 2] proved that if an operator $T$ on a Hilbert space is such that $T^{*}=S^{-1} T S$ and $0 \notin \mathrm{cl}(W(S))$ then $T$ is similar to a selfadjoint operator. He further showed with different techniques that under this hypothesis on $T$, the spectrum of $T$ lies on the real line. Taking a clue from this theorem, U.N. Singh and Kanta Mangla [8] obtained the following result: If $T$ is a nonsingular operator such that $T^{*}=S^{-1} T^{-1} S$ and $0 \notin \mathrm{cl}(W(S))$, then $T$ is similar to a unitary operator. So in particular $\sigma(T)$ lies on the unit circle. The author [7] has recently proved the following result using the technique of Williams [10, Theorem 1] which includes this particular case: If $T$ is an operator such that for some integers $p, q$, $T^{* p}=S^{-1} T^{q} S$ and $0 \notin \operatorname{cl}(W(S))$ then for $\lambda \in \sigma(T), \lambda^{* p}=\lambda^{q}$. In the present note our interest is to develop these ideas when the canonical image of $T^{* p}$ in the Calkin algebra is similar to that of $T^{q}$ for some integers $p, q$.

We shall suppose all operators are defined on an infinite dimensional separable Hilbert space $H$. For an operator $T$, we write $\sigma(T)$ for the spectrum, Bdry $\sigma(T)$ for the boundary of $\sigma(T), \pi_{00}(T)$ for the isolated points of $\sigma(T)$ that are eigenvalues of finite multiplicity and $\operatorname{cl}(W(T))$ for the closure of the numerical range. If $\hat{T}$ is the canonical image of $T$ in the Calkin algebra (the algebra of all operators modulo the ideal of compact operators), then $\sigma(\hat{T})$ will be called the essential spectrum of $T$. By the left essential spectrum of $T, \sigma_{1}(\hat{T})$, we mean the collection of all $\lambda$ 's such that $\hat{T}-\lambda I$ fails to be left-regular. The numerical range $W_{e}(\hat{T})$ of $\hat{T}$ is called the essential numerical range of $T$. As shown in [9, Theorem 9],

Received by the editors September 18, 1972 and, in revised form, March 23, 1973. AMS (MOS) subject classifications (1970). Primary 47A10, 47B20.

Key words and phrases. Hilbert space, operator, spectrum, numerical range, canonical image, cramped unitary operator, compact operator.

(c) American Mathematical Society 1974 
$W_{e}(\hat{T})=\bigcap_{K} \operatorname{cl}(W(T+K))$, where the intersection is taken over all compact operators $K$.

Throughout the note, $K$ and $K_{n}$ 's will be compact operators and $p, q$ will denote some integers.

For the proof of our results we shall need the following lemmas.

LEMMA 1 [4]. A complex number $\lambda \in \sigma_{1}(\hat{T})$ if and only if there exists $a$ sequence $\left\{x_{n}\right\}$ of unit vectors in $H$ such that $x_{n} \rightarrow 0$ weakly and $\left\|(T-\lambda) x_{n}\right\| \rightarrow$ 0 .

Lemma 2 [6, Theorem 1]. Bdry $\sigma(T) \subseteq \sigma_{1}(\hat{T}) \cup \pi_{00}(T)$.

Our main result is the following

THEOREM. If $T$ is an operator such that $S T^{* p}=T^{q} S+K$ and $0 \notin W_{e}(\hat{S})$, then for any $\lambda \in \sigma(\hat{T}), \lambda^{* p}=\lambda^{q}$.

Proof. Since $0 \notin W_{e}(S)$, there exists a compact operator $K$ such that $0 \notin \operatorname{cl}(W(S+K))$. Therefore we assume $0 \notin \operatorname{cl}(W(S))$ without any loss of generality. Now $\sigma(\hat{T})=\sigma_{1}(\hat{T}) \cup \sigma_{1}\left(\hat{T}^{*}\right)^{*}$. Therefore by the reason of symmetry it suffices to show that if $\lambda \in \sigma_{1}(\hat{T})$, then $\lambda^{* p}=\lambda^{q}$. By Lemma 1 , for $\lambda \in \sigma_{1}(\hat{T})$, there exists a sequence $\left\{x_{n}\right\}$ of unit vectors such that $x_{n} \rightarrow 0$ weakly and $\left\|(T-\lambda) x_{n}\right\| \rightarrow 0$. Then

$$
\begin{aligned}
\mid\left(\lambda^{* p}\right. & \left.-\lambda^{q}\right)\left\langle S^{-1} x_{n}, x_{n}\right\rangle \mid \\
& =\left|\left\langle\left(\lambda^{* p}-T^{* p}+S^{-1} T^{q} S+K-\lambda^{q}\right) S^{-1} x_{n}, x_{n}\right\rangle\right| \\
& =\left|\left\langle\left(\lambda^{* p}-T^{* p}\right) S^{-1} x_{n}, x_{n}\right\rangle+\left\langle S^{-1}\left(T^{q}-\lambda^{q}\right) x_{n}, x_{n}\right\rangle+\left\langle K S^{-1} x_{n}, x_{n}\right\rangle\right| \\
& =\left|\left\langle S^{-1} x_{n},\left(\lambda^{p}-T^{p}\right) x_{n}\right\rangle+\left\langle S^{-1}\left(T^{q}-\lambda^{q}\right) x_{n}, x_{n}\right\rangle+\left\langle S^{-1} x_{n}, K^{*} x_{n}\right\rangle\right| \\
& \leqq\left\|S^{-1}\right\|\left\{\left\|\left(T^{p}-\lambda^{p}\right) x_{n}\right\|+\left\|\left(T^{q}-\lambda^{q}\right) x_{n}\right\|+\left\|K^{*} x_{n}\right\|\right\} .
\end{aligned}
$$

Since $x_{n} \rightarrow 0$ weakly, the compactness of $K^{*}$ implies $\left\|K^{*} x_{n}\right\| \rightarrow 0$. Also, it is easy to verify that $\left\|\left(T^{p}-\lambda^{p}\right) x_{n}\right\| \rightarrow 0$ and $\left\|\left(T^{q}-\lambda^{q}\right) x_{n}\right\| \rightarrow 0$. Thus $\left|\left(\lambda^{* p}-\lambda^{q}\right)\left\langle S^{-1} x_{n}, x_{n}\right\rangle\right| \rightarrow 0$. Since $0 \notin \operatorname{cl}\left(W\left(S^{-1}\right)\right)$, it follows that $\lambda^{* p}=\lambda^{a}$. This finishes the proof of our theorem.

The following corollary is essentially the same due to V. Istratescu and I. Istratescu [5, Theorem 2.1].

COROLlaRY 1. If $T$ is an operator for which $S T^{*}=T S+K$ and $0 \notin W_{e}(\hat{S})$ then

1. if $\pi_{00}(T)=\varnothing$, then $\sigma(T) \subseteq R$, where $R$ denotes the real line;

2. the set $\sigma(T) \cap R^{\prime}$, where $R^{\prime}$ is the complement of $R$, is at most countable.

Proof. First we consider Case 1 . Then by Lemma 2 Bdry $\sigma(T) \subseteq \sigma_{1}(\hat{T})$. If $\lambda \in \sigma_{1}(\hat{T})$ then by the hypothesis $\lambda^{*}=\lambda$. Thus Bdry $\sigma(T)$, and hence $\sigma(T)$, is real. 
Next we prove Case 2. Since $\sigma_{1}(\hat{T})$ is real and $\pi_{00}(T)$ is at most countable (because the set of isolated points of $\sigma(T)$ is at most countable), it follows by Lemma 2 that Bdry $\sigma(T) \cap R^{\prime}$, and hence $\sigma(T) \cap R^{\prime}$, is finite or countable.

Our next corollary is parallel to Corollary 1.

COROLlaRY 2. If $T$ is a nonsingular operator such that $S T^{* p}=T^{q} S+K$ and $0 \notin W_{e}(S)$, where $p \neq q$, then

1. if $\pi_{00}(T)=\varnothing$, then $\sigma(T) \subseteq \Gamma$, where $\Gamma$ denotes the unit circle;

2. the set $\sigma(T) \cap \Gamma^{\prime}$, where $\Gamma^{\prime}$ is the complement of $\Gamma$, is at most countable.

Proof. If $\lambda \in \sigma_{1}(\hat{T})$, then $\lambda^{* p}=\lambda^{q}$, and so $|\lambda|^{p}=|\lambda|^{q}$. Since $\lambda \neq 0$ as $T$ is nonsingular, and $p \neq q$, we have $|\lambda|=1$. If $\pi_{00}(T)=\varnothing$, then by Lemma 2 , Bdry $\sigma(T) \subseteq \Gamma$. Consequently, any $\lambda$ in $\sigma(T)$ with maximum or minimum absolute value belongs to $\Gamma$. Hence $\sigma(T) \subseteq \Gamma$. This proves Case 1. Next again by Lemma 2, it is easy to see that Bdry $\sigma(T) \cap \Gamma^{\prime}$ and Bdry $\sigma\left(T^{-1}\right) \cap$ $\Gamma^{\prime}$ are at most countable. Consequently, Case 2 follows.

The above corollary can be established with an alternate approach in case $p=-q$. For the sake of convenience we assume $p=1$. As we have seen in the proof of our theorem, we assume $0 \notin \mathrm{cl}(W(S))$. Then we can separate 0 from $\mathrm{cl}(W(S))$ by a half plane, by replacing $S$ by $S e^{i \theta}$ if necessary. Let us choose $\theta$ so that this half plane lies strictly to the right of the imaginary axis. Let $A=\left(S+S^{*}\right) / 2$. Then $A$ is a positive nonsingular operator. Let $A^{1 / 2}$ be the square root of $A$ which is also positive nonsingular. Now, by our hypothesis, $S T^{*}=T^{-1} S+K_{1}$ and $S^{*} T^{*}=T^{-1} S^{*}+K_{2}$. Then $A T^{*}=T^{-1} A+$ $K_{3}$. Substituting $T_{1}=A^{-1 / 2} T A^{1 / 2}$, we have $T_{1}^{*}=T_{1}^{-1}+K_{4}$, or $T_{1}^{*} T_{1}=I+K_{5}$. Consequently, $\left(T_{1}^{*} T_{1}\right)^{1 / 2}=I+K_{6}$. Now $T_{1}=U P$ where $U$ is unitary and $p=$ $\left(T_{1}^{*} T_{1}\right)^{1 / 2}$. It follows in turn that $T_{1}=U+K_{7}$. This shows that $T$ is similar to the sum of a unitary and a compact operator. Now, one can easily verify Case 1 and Case 2.

COROLlaRY 3. If $T$ is an operator such that $S T^{* p}=T^{q} S+K$ and $0 \notin W_{e}(\hat{S})$ where $|p| \neq|q|$, then $\sigma(T)$ is at most countable.

Proof. Since $\lambda^{* p}=\lambda^{a}$ for $\lambda \in \sigma_{1}(\hat{T})$, it follows that $|\lambda|=1$ if $\lambda \neq 0$. As $|p| \neq|q|$, we have $\lambda^{p+q}=1$, which shows that $\sigma_{1}(\hat{T})$ is finite. Again by Lemma 2 , Bdry $\sigma(T)$ is at,most countable and hence so is $\sigma(T)$.

Next we wish to obtain some results using the previous ones. An operator $T$ is said to satisfy condition $-\left(G_{1}\right)$ if $(T-\lambda)^{-1}$ is normaloid for all $\lambda \notin \sigma(T)$; if every direct summand of $T$ satisfies the condition $-\left(G_{1}\right)$, then $T$ is called reduction $-\left(G_{1}\right)$.

Corollary 4. If $T$ is a reduction $-\left(G_{1}\right)$ operator for which $S T^{*}=$ $T^{p} S+K$ and $0 \notin W_{e}(\hat{S})$, then $T$ is normal. 
Proof. If $|p| \neq 1$, then $\sigma(T)$ is at most countable by Corollary 3 . Therefore in this case Theorem 1 of [1] is applicable. For $p=1, \sigma(T) \cap R^{\prime}$ is at most countable by Corollary 1. Using Theorem 4.1 of [5], $T$ turns out to be normal. Lastly, let us assume $p=-1$.

Let $M$ be the closed linear span of all reducing eigenspaces of $T$. Then the restriction of $T$ to $M$, denoted by $T_{1}=T / M$ is normal. Let $T_{2}=T / M^{\perp}$, where $M^{\perp}$ denotes the orthogonal complement of $M$. Then $T=T_{1} \oplus T_{2}$. We assert that $T_{2}$ is unitary; whence it will follow that $T$ is normal.

First of all we claim $\pi_{00}\left(T_{2}\right)=\varnothing$. If $\pi_{00}\left(T_{2}\right) \neq \varnothing$, then for $\lambda \in \pi_{00}\left(T_{2}\right)$, $\operatorname{Ker}\left(T_{2}-\lambda I\right)=\operatorname{Ker}\left(T_{2}^{*}-\lambda^{*} 1\right)$ (see [3, Example 6]). But by construction, $\operatorname{Ker}(T-\lambda) \subseteq M^{\perp}, \operatorname{Ker}\left(T_{2}-\lambda I\right)=\operatorname{Ker}(T-\lambda I)$ [2, Proposition 4.1] and $\operatorname{Ker}\left(T_{2}^{*}-\lambda^{*} I\right) \subseteq \operatorname{Ker}\left(T^{*}-\lambda^{*} I\right)$. Therefore, $\operatorname{Ker}(T-\lambda I) \subseteq \operatorname{Ker}\left(T^{*}-\lambda^{*} I\right)$. It follows from this relation that $\operatorname{Ker}(T-\lambda I)$ reduces $T$ and hence $\operatorname{Ker}(T-\lambda I) \subseteq M$, a contradiction. Therefore $\pi_{00}\left(T_{2}\right)=\varnothing$. Since $p=-1$, it follows by our main result that Bdry $\sigma\left(T_{2}\right) \subseteq \sigma_{1}\left(\hat{T}_{2}\right) \subseteq \sigma_{1}(\hat{T}) \subseteq \Gamma$. As argued before we get $\sigma\left(T_{2}\right) \subseteq \Gamma$. Since $T_{2}$ is reduction $-\left(G_{1}\right), T_{2}$ turns out to be unitary. This completes the proof.

In [8], it is shown that if $T$ is a nonsingular operator such that $T^{*}=$ $U^{*} T^{-1} U$, where $U$ is a cramped unitary operator, then $T$ is unitary. Here we wish to obtain a parallel result.

COROLlARY 5. If $T$ is a nonsingular operator such that $U T^{*}=T^{-1} U+K$ where $U$ is an operator such that $\hat{U}$ is unitary and $0 \notin W_{e}(\theta)$, then $T$ is the sum of a unitary and a compact operator.

Proof. Since $O$ is unitary, $U^{*} U-1$ and $U U^{*}-I$ are both compact. Then, by our hypothesis $\mid T^{*}=U^{*} T^{-1} U+K_{1}$ and $T=U^{*} T^{*-1} U+K_{1}^{*}$, leading to $T^{*} T=U^{*}\left(T^{*} T\right)^{-1} U+K_{2}$. Applying our main result, we get $\sigma\left(T^{*} T\right)^{\wedge} \subseteq \Gamma$. Since $T^{*} T$ is a positive nonsingular operator, we have $\sigma\left(T^{*} T\right)^{\wedge}=\{1\}$. As argued in the alternate proof of Corollary 2, we arrive at the required conclusion.

The author wishes to thank Dr. B. S. Yadav and also the referee for their valuable suggestions to improve the original text of the paper.

\section{REFERENCES}

1. S. K. Berberian, Some conditions on an operator implying normality. II, Proc. Amer. Math. Soc. 26 (1970), 227-281. MR 42 \#884.

2. - The Weyl spectrum of an operator, Indiana Univ. Math. J. 20 (1970) (71), 529-544. MR 43 \#5344.

3. - An extension of Weyl's theorem to a class of not necessarily normal operators, Michigan Math. J. 16 (1969), 273-279. MR 40 \#3335.

4. P. A. Fillmore, J. G. Stampfli and J. P. Williams, On the essential numerical range, the essential spectrum and a problem of Halmos, Acta. Sci. Math. (Szeged) 33 (1972), 179-192. 
5. V. Istratescu and I. Istrațescu, On some classes of operators. II, Math. Ann. 194 (1971), 126-134. MR 44 \#7351.

6. C. R. Putnam, The spectra of operators having resolvents of first order growth, Trans. Amer. Math. Soc. 133 (1968), 505-510. MR 37 \#4651.

7. S. M. Patel, On powers of an operator similar to those of its adjoints, Rev. Roumaine Math. Pures Appl. (to appear).

8. U. N. Singh and Kanta Mangla, Operators with inverses similar to their adjoints, Proc. Amer. Math. Soc. 38 (1973), 258-260.

9. J. G. Stampfli and J. P. Williams, Growth conditions and the numerical range in Banach algebra, Tôhoku Math. J. (2) 20 (1968), 417-424. MR 39 \#4674.

10. J. P. Williams, Operators similar to their adjoints, Proc. Amer. Math. Soc. 20 (1969), 121-123. MR 38 \#1552.

Faculty of Mathematics, University of Delhi, Delhi-7, India 